\title{
The Light emitted from Solidified Gases and its Relation to Cosmic Phenomena.
}

\author{
By Prof. L. VEgard, University of Kristiania.
}

$\mathrm{C}^{\mathrm{N}}$ previous communications ${ }^{1} \mathrm{I}$ have given accounts of investigations on light emitted from solid nitrogen exposed to cathode rays and its bearing on the auroral spectrum. The conclusion was reached that in the auroral region the temperature was so low that nitrogen existed partly in the form of minute crystalline particles. If these particles are of molecular order of magnitude, the state is very much like a gas, from which, however, it differs in one respect. The constituent particles are not molecules with identical mass, but each particle may contain a more or less arbitrary number of atoms arranged in a space lattice characteristic of the solid state. This state, which is something between a gas and a solid, we shall call a pseudogaseous state.

Although it is difficult or perhaps impossible to produce this pseudo-gaseous state in laboratory experiments, still I was able to show that a layer of solid nitrogen when bombarded with cathode rays of small velocity gave a spectrum which was essentially the same as the auroral spectrum. In the green, the spectrum from solid nitrogen consisted of two bands, $\mathrm{N}_{1}$ and $\mathrm{N}_{2}$, corresponding to the auroral lines 5577 and $5^{2} 30$, and in addition it showed in the blue and violet the band-heads which are typical for the auroral spectrum.

The work has been continued at the Cryogenic Laboratory of Leyden and I have been able to study the new effect under varied conditions, with different kinds of rays, and I have also experimented on layers of various gases. In order to determine more exactly the position and structure of the bands, I have used a spectrograph of considerable dispersion. With the largest dispersion used, the distance between the yellow helium line $\left(5^{875} \cdot 6\right)$ and the green helium line $\left(5 \circ 5^{\circ} \cdot 7\right)$ was $27 \mathrm{~mm}$.

I have obtained about a hundred spectrograms of the light effect from solidified gases and made a number of interesting observations which will be dealt with in greater detail in a later work. In this article I propose to give a short and preliminary account of some of my recent results.

Already in my first publication I mentioned that while the line $\mathrm{N}_{2}$ was fairly sharp, $\mathrm{N}_{1}$ was broad and covered the spectral region $55^{2} 5-5660$, and further it showed some structure. The spectrograms at larger dispersion ${ }^{2}$ showed that $\mathrm{N}_{1}$ had three maxima, one strong and two weak.

The position of the maxima varies somewhat with the experimental conditions. From one of my spectrograms with large dispersion $I$ find that $N_{1}$ covers a region between $55^{28}$ and 5655 . For the principal maximum I find $\lambda=5555$, and for the two weak ones $5^{6 \mathrm{II}}$ and 5649. Also with the greater dispersion, $\mathrm{N}_{2}$ appeared as a single but somewhat diffuse line with a wave-length $5^{229} 4$, which agrees very well with the wave-length (5230) given in my first publications.

So long as we are unable to experiment on a pseudogaseous state of solid nitrogen, we cannot make an

1 Proc. Roy. Akad. Amsterdam, vol. xxvii. Nos. I and 2 ; Nature, vol.

Ir3, No. 2846 , p. 7r6; C.R. Acad. Sc. t. r78, p. Ir53. exact comparison between the wave-length of $\mathrm{N}_{1}$ and that of the prominent green auroral line. Under the conditions of the experiments, $N_{1}$ does not define any definite wave-length, and we can merely state that $N_{1}$ is extending on both sides of the auroral line. The sharpness of the latter must be due to the smallness of the particles, and the prominent green auroral line is to be regarded as the limiting aspect of the band $N_{1}$, when emitted from solid nitrogen particles of molecular order of magnitude, which exist in free space.

I have been able to make some experiments which actually show that the bands get sharper when the particles are reduced in size. When an inert gas which contains traces of nitrogen is condensed on the cold surface, the layer will contain small nitrogen particles surrounded by an inert substance. In this state, we should expect nitrogen to behave in a similar way as if the particles existed in free space. Experiments were carried out in mixtures of argon and nitrogen. In one series I used canal rays, in another swift cathode rays ; but in all cases $I$ found that the $N_{1}$ band became narrower when the concentration of nitrogen became smaller.

A.t the same time I observed the remarkable fact that the position of the principal maximum changes with the nitrogen concentration. For small concentrations the principal maximum approaches a wave-length 5604 . Thus with varying composition of the mixture the principal maximum oscillates between the values 5555 and 5604. The "secondary" maxima seem to disappear when the concentration of nitrogen is very small.

The motion of the principal maximum approximately follows the relation

$$
\lambda_{0}-\lambda_{p}=\frac{p}{\mathrm{rOO}}\left(\lambda_{0}-\lambda_{100}\right)
$$

where $\lambda_{p}$ is the wave-length corresponding to the percentage $p$ of nitrogen and $\lambda_{0}$ and $\lambda_{100}$ are the limiting positions.

The motion of the band may be due partly to the change of the average size of the nitrogen particles and partly to a mutual influence between argon and nitrogen. Perhaps an X-ray analysis of the crystalline mixture would give some information regarding this point. At any rate, the experiments indicate that a reduction of the size of the particles makes the band sharper, and that the position of the line corresponding to a pseudo-gaseous state need not coincide with any of the maxima observed in ordinary nitrogen under the conditions of my experiments.

The contraction of the bands does not seem to be essentially effected by change of temperature. By working under reduced pressure with liquid hydrogen, the temperature of the layer was lowered from $20^{\circ}$ to $\mathrm{r} 4^{\circ} \mathrm{K}$.; but there was no contraction of $\mathrm{N}_{1}$ to be observed, although a change of a few per cent. would have been detected.

The experiments in argon mixtures also give an interesting illustration of the extremely high emission power of solid nitrogen. Even very "pure" argon contained sufficient nitrogen to give a marked emission of $\mathrm{N}_{1}$ and $\mathrm{N}_{2}$; and the nitrogen afterglow. The addition NO. 2862 , VOL. I I 4$]$ 
of about o I per cent. of nitrogen to the "pure " argon considerably increased the nitrogen effect.

As shown in my earlier communications, slowly moving cathode rays gave in the blue and violet just the same negative and positive band-heads as those typical for the auroral spectrum. The effect has since been photographed with a quartz spectrograph through a quartz window, and the spectrum in the ultraviolet showed the same positive band-heads as those observed in the auroræ.

When the layer is bombarded with a powerful beam of cathode rays from a Wehnelt cathode, a deep red luminosity appears near the layer, and the colour has the same appearance as a red colour very characteristic of the auroræ. A spectrum obtained on a panchromatic plate showed the same two red bands which I have obtained on auroral spectrograms. Thus from the system I am experimenting upon I get the characteristic bands and lines of the auroral spectrum from red to ultraviolet.

Now it is of great importance to see whether also positive rays might give the typical auroral spectrum. For this purpose a considerable number of experiments have been made with canal rays acting on nitrogen, hydrogen, and helium.

Exposed to canal rays, solid nitrogen emitted both $N_{1}$ and $N_{2}$; but in the blue, violet, and ultraviolet, the canal-ray spectrum was quite different from the typical auroral spectrum. In this spectral region the canalray spectrum was dominated by a series of diffuse bands, which extended far into the ultraviolet. Moreover, as has been shown before, the canal-ray spectrum has a number of strong lines not present in the auroral spectrum.

Thus neither in the solid nor in the gaseous state of nitrogen do the canal rays produce the nitrogen spectrum observed in the auroræ, and hence we conclude that by far the greater part of the auroræ must be caused by cathode rays.

Cathode rays which were produced by an induction coil and had a fairly large velocity corresponding to a spark-gap of 5-6 mm. between spheres I cm. diameter, gave a very brilliant effect, showing $\mathrm{N}_{1}$ and $\mathrm{N}_{2}$ very strongly. Under these conditions I also obtained the same series of diffuse bands as were obtained with canal rays, but their intensities were now relatively smaller. ${ }^{3}$

Nitrogen-argon mixtures were investigated both with canal rays and swift cathode rays, and some results with regard to the variations of $\mathrm{N}_{1}$ have already been described. Not only $\mathrm{N}_{1}$, but also the diffuse canal-ray bands in blue and violet, were seen to contract as the concentration of nitrogen became smaller, and at the same time each maximum moved towards shorter wave-lengths. When the layer of argon only contained small quantities of nitrogen, the bands were concentrated into fairly well-defined diffuse lines. In the blue and violet there were two bands, each of which was concentrated into one pair of lines, one pair at $\lambda=45^{2} 3,4473$, and the second at $\lambda=4236,42 \mathrm{r} x$.

These bands are no doubt one state of development of the two bands observed by Lord Rayleigh in the light from the night sky. This means that this light should be produced by fairly slow positive rays.

3 It remains still to be shown that the bands in blue-ultraviolet are not produced by canal rays, either retrograde rays or rays produced by imperfect rectification of the current.
When the layer of solid nitrogen has been bombarded for some time with canal rays or swift cathode rays, the afterglow becomes very persistent. Two or three minutes after the rays are cut off the light intensity from the layer may remain practically constant for hours or perhaps for days. Usually the afterglow only shows the band $\mathrm{N}_{2}$; but when the persistent afterglow is very strong, I have also observed $\mathrm{N}_{1}$ and the bands in the blue.

When the liquid hydrogen disappears and the temperature of the layer gradually increases, the stored energy is suddenly released under intense light emission, showing $\mathrm{N}_{2}$ very strongly, but also $\mathrm{N}_{1}$ and the diffuse bands in the blue appear very strong. Thus $\mathrm{N}_{1}$ and $\mathrm{N}_{2}$ and the series of diffuse bands in the blue-ultraviolet are emitted in the afterglow and consequently from the very interior of the solid substance.

When the temperature of the layer increases to a certain point the appearance of the solid nitrogen suddenly changes. At the temperature of liquid hydrogen the layer resembles a piece of ice and sticks to the cold surface; but at a certain point the layer assumes a powdery form, and if it is somewhat thick it falls down from the cold copper surface. The existence of two modifications of solid nitrogen was discovered some years ago by means of cooling curves, ${ }^{4}$ and the transformation is found to take place at a temperature of $35.5^{\circ} \mathrm{K}$.

Now the energy stored in the afterglow radiates out at the very moment that the layer passes over into the other modification. The phosphorescent energy cannot exist in the modification which is stable above $35.5^{\circ} \mathrm{K}$. This phenomenon is explained from the fact that the light given out by the afterglow is characteristic of the modification of solid nitrogen which is stable below $35.5^{\circ} \mathrm{K}$., for if nitrogen in the other modification is exposed to cathode or canal rays, $\mathrm{N}_{1}, \mathrm{~N}_{2}$, and the diffuse bands in the blue-violet are not emitted. The luminosity, which is very faint, shows a different spectrum and no afterglow.

This fact has a very important bearing on cosmic phenomena. In that part of the atmosphere where $\mathrm{N}_{1}$ and $\mathrm{N}_{2}$ are emitted the temperature must be below $35.5^{\circ} \mathrm{K}$., and the isothermal surface $35.5^{\circ} \mathrm{K}$. marks the transition between an upper layer very easily excited to light emission and a lower layer which has lost this high emission power.

In this way I think we get the explanation of the fact found by Lindemann that the meteor frequency as a function of altitude shows two maxima with a very marked minimum in between, for below the isothermal surface $\left(35^{\circ} 5^{\circ}\right)$ the meteors may often seem to disappear because they produce very little light until farther down they become luminous on account of heating. Now the height of this isothermal surface ought to be greater in the day than in the night, greater at the equator than at the poles, and greater in the summer than in the winter. Indeed, Lindemann has found that the height of the upper frequency maximum, caused by nitrogen below $35.5^{\circ} \mathrm{K}$., increases from $75 \mathrm{~km}$. in the winter to $85 \mathrm{~km}$. in the summer.

The relative intensity of the lines $\mathrm{N}_{1}$ and $\mathrm{N}_{2}$ is of importance not only from a physical point of view, but also in connexion with the auroral problem. The 4 W. H. Keesom and H. Kamerlingh Onnes, Proc. Roy. Akad. Amsterdam, 24, p. I3I5.

NO. 2862 , VOL. I I 4$]$ 
intensity ratio $\mathrm{N}_{1} / \mathrm{N}_{2}$ increases generally with velocity and the density of the bombarding electric rays, and in nitrogen-argon mixtures it increases very much when the nitrogen concentration diminishes. With very small concentrations $\mathrm{N}_{\mathbf{1}}$ predominates as compared with $\mathrm{N}_{2}$. This effect indicates that $\mathrm{N}_{1} / \mathrm{N}_{2}$ increases as the nitrogen particles get smaller, and it may thus account for the predominance of the auroral line 5577 in the auroral spectrum.

The great variability of $\mathrm{N}_{1} / \mathrm{N}_{2}$ makes it difficult to determine exactly the minimum cathode ray velocity necessary for producing the two bands. Perhaps they require the same velocity, only that the intensity of $\mathrm{N}_{1}$ usually is very small at small velocities. At the present time, I have been able to follow $\mathrm{N}_{2}$ down to velocities corresponding to 78 volts, and $\mathrm{N}_{1}$ down to I65 volts.

A non-luminous layer of solid nitrogen was not made luminous by ultraviolet light from a condensed aluminium spark. X-rays, however, produced the ordinary afterglow.

When the layer is in the state showing the persistent afterglow, conditions are different. In this case light from an ordinary incandescent lamp with a tungsten filament produced a very marked increase in the intensity of the afterglow. In some cases the intensity underwent very marked periodic variations with periods of 5-6 seconds, and I could count 8-9 periods.

Layers of solid oxygen, ammonia, and argon have been bombarded with both cathode rays and canal rays, and a layer of nitrous oxide with canal rays only. With the exception of argon, the light effect was feeble and gave no afterglow. The fairly strong effect observed from an argon layer was to a great extent due to traces of nitrogen, which produced a weak afterglow and a strong emission of $N_{1}$ and the canal-ray bands of nitrogen. The afterglow showed only the line $\mathrm{N}_{2}$ and no other visible light in the spectrum. Thus argon itself gives no afterglow, and the real argon effect, although somewhat stronger, perhaps, than in the case of oxygen ammonia and nitrous oxide, is an effect of the same type as shown by these gases.

The experiments on layers of various substances thus show that the light effect from nitrogen at temperatures below $35.5^{\circ}$, which is applicable to the auroral spectrum, is a light effect of a unique and singular type.

\section{The Beam System of Radio Telegraphy.}

THE paper read by Senator Marconi to the Royal Society of Arts on July 2, and published in the Journal of the Society for July 25, describing the results he has obtained over very long distances by short wave directional radio telegraphy, is one of great importance and marks an epoch in the development of long distance radio communication. Mr. Marconi reminds us that more than twenty-eight years ago he showed Sir William Preece the transmission and reception of signals over a distance of nearly two miles by a "beam" system, using short waves and reflectors. Curiously enough, at that time he could only transmit signals by means of an antenna over a

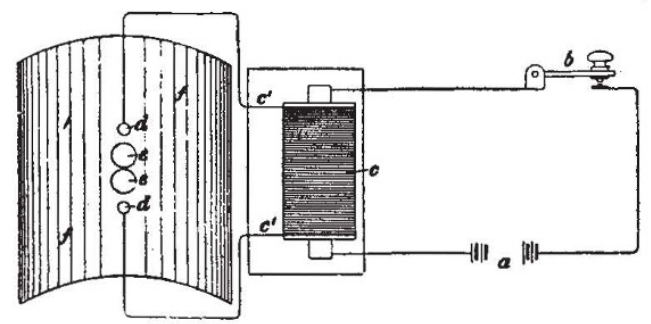

Fig. I. - Spark transmitter and sheet metal reflector, 1896.

distance of half a mile. The rapid and spectacular progress made, however, by the long wave system in those early days diverted attention from short wave research, although the latter could be emitted in beams in definite directions.

In 1899 , Mr. Marconi read a paper to the Institution of Electrical Engineers in which he pointed out that a short wave system would be of value in connexion with lighthouses and lightships in foggy weather, enabling them to locate dangerous points. He also projected a beam of waves across the lecture room, a bell ringing only when the reflector faced the receiver (Figs. I and 2).

In I9r6, in conjunction with Mr. C. S. Franklin, he again began to make researches with directive beams. The reflectors used at first were not solid sheets of metal, but consisted of wires forming a cylindrical parabolic reflector, the antenna forming the focal line of the cylinder (Fig. 3). The reflectors now used consist of a grid of antennæ and a grid of reflecting wires arranged parallel to one another (Fig. 4). Special arrangements have to be made so as to ensure that the phase of the oscillations in all the wires is the same. The great value of reflectors was demonstrated by experiment, the energy received being increased about two hundredfold by their use.

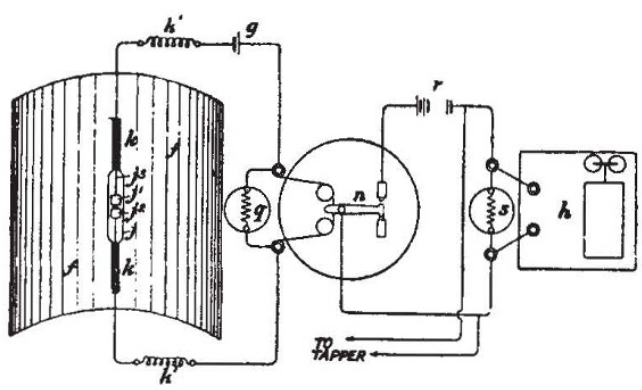

Fig. 2,-Coherer receiver and sheet metal reflector, 1896.

Many tests were carried out between the steam yacht Elettra and a small station erected at Poldhu which emitted waves about roo metres in length, the frequency therefore being about $3,000,000$. One definite result obtained was that the strength of the signals received during the hours of daylight varied inversely with the mean altitude of the sun over the space intervening between the two stations. It is wrong, therefore, to refer to distances covered during daylight as "day ranges." It shows also that the tests which were carried out partly within the tropics during the months of May and June were made under very unfavourable conditions.

NO. 2862 , VOL. II 4 ] 